and distribution of these factors was assessed between the five groups. Logistic regression was used to assess the association between patient characteristics and in-hospital survival after an RTI.

Results A total of 94316 patients were included in this study. Only $11 \%$ were women and $74 \%$ of the sample was between $15-45$ years of age. Most of the injuries (96\%) had an ISS between 1 and 15 and in-hospital survival was $97.4 \%$. Survival was strongly associated with hospital of presentation (AOR 4.4, CI 3.4 to 5.7). Other significant predictors of survival included mode of arrival (AOR 1.5, CI 1.2 to 1.8) and age (AOR 0.16, CI 0.09 to 0.26 ) after adjustment for severity and type of injury.

Significance The analysis shows a strong association between centre and survival even after adjustment for type and severity of injury and other important confounders. This indicates differences in quality of trauma care within hospitals in Karachi requiring further in-depth studies for healthcare policy change.

\title{
37 DIFFERENCES IN RISK-ADJUSTED OUTCOME OF ROAD TRAFFIC INJURIES IN URBAN TERTIARY CARE CENTRES OF PAKISTAN: ANALYSIS OF SURVEILLANCE DATA
}

doi:10.1136/injuryprev-2012-040590w.37

${ }^{1} \mathrm{~A}$ Mehmood, 'J Razzak, 'U Mir, ${ }^{2} \mathrm{R}$ Jooma. ${ }^{1}$ Aga Khan University, Karachi, Pakistan;

${ }^{2}$ Road Traffic Injury Research and Prevention Centre, JPMC, Karachi, Pakistan

Background Trauma due to road traffic injuries (RTIs) poses a considerable burden on the healthcare system. Outcome of trauma varies between countries, within countries and between different trauma centres. This variation reflects the potential for improvement in trauma care.

Aims and Objectives To assess the difference in RTI trauma survival among five tertiary care institutes in Karachi, Pakistan.

Methods All cases registered in the RTI surveillance system (operational in five major tertiary care hospitals in Karachi) between September 2006 and September 2009 were included. Data on the patients' age, sex, mode and delay in arrival, type and severity of injury were extracted. Data were stratified by hospital of presentation 